\title{
Biological Template Based on ent-Kaurane Diterpenoid Glycosides for the Synthesis of Inorganic Porous Materials
}

\author{
Ángela B. Sifontes ${ }^{1 *}$, Mirla Rodriguez ${ }^{1}$, David Freire ${ }^{1}$, Wendy Rondón ${ }^{1}$, Ligia Llovera ${ }^{1}$, \\ Edgar Cañizales ${ }^{2}$, Franklin J. Méndez ${ }^{1}$, Andrea Monaco ${ }^{1}$, Yraida Díaz ${ }^{1}$ \\ ${ }^{1}$ Centro de Química, Instituto Venezolano de Investigaciones Científicas, Caracas, Venezuela \\ ${ }^{2}$ PDVSA, Intevep, Los Teques, Venezuela \\ Email: *angelasifontes@gmail.com, asifonte@ivic.gob.ve
}

Received August 24, 2013; revised September 26, 2013; accepted October 3, 2013

Copyright (C) 2013 Ángela B. Sifontes et al. This is an open access article distributed under the Creative Commons Attribution License, which permits unrestricted use, distribution, and reproduction in any medium, provided the original work is properly cited.

\begin{abstract}
Recent studies on the preparation of porous nano-materials revealed that the use of kaurane diterpenoids molecules from steviol as biological template favors the obtaining of metallic oxides with tubular morphology as nanorods or nanofibers. In this sense, the present contribution shows an analysis in order to understand how these glycosides of kaurane diterpenoids control the nucleation and growth of inorganic materials favoring the obtaining of these morphologies. For this purpose, it was necessary to carry out studies of the leaf aqueous extract of Stevia rebaudiana by HRTEM, FTIR and ${ }^{1} \mathrm{H}-\mathrm{NMR}$.
\end{abstract}

Keywords: Diterpene Glycosides; Stevia rebaudiana; Template

\section{Introduction}

Diterpenoids constitute a class of natural compounds among the richest in terms of chemical structure and highly diverse properties [1-3]. Diterpenoids can be subdivided into acyclic (phytanes), bicyclic (labdanes, clerodanes), tricyclic (pimaranes, abietanes, cassanes, rosanes, vouacapanes, podocarpanes), tetracyclic (trachylobanes, kauranes, aphidicolanes, stemodanes, stemaranes, beyeranes, atisan macrocy-clicditerpernes (taxanes, cembranes, daphnanes, tiglianes, ingenanes) and mixed compounds, in accordance with the number and the cyclization patterns displayed by their skeletal $[1,2]$. Natural compounds of this class have been found in many plants, fungi, sea organisms and insects [1]. In particular, the kauranes represent an important group of tetracyclic diterpenes and their structures are constituted by a perhydrophenantrene unit ( $a, b$ and $c$ rings) fused with a cyclopentane unit ( $d$ ring) formed by a bridge of two carbons between C-8 and C-13 (Figure 1) [2]. Several criteria are used for the nomenclature of the kaurane diterpenes, the most frequent being the inversion of the conventional description of stereochemistry when the name is preceded by the prefix "ent-" [1,4-6].

"Corresponding author.
Recently, glycosides of the kaurane diterpene known as steviol (ent-13-hydroxykaur-16-en-19-oic acid) have been employed as biological templates in the preparation of porous nano-materials $[4,7]$. In this sense, the steviol is a constituent from Stevia rebaudiana, a plant native to certain regions of South America (Paraguay and Brazil). The major constituents in the leaves of $S$. rebaudiana are the potently sweet diterpenoid glycosides stevioside, rebaudiosides A and D and dulcoside A (Figure 2). The steviol glycoside preparations could be obtained by hot-water extraction from the leaves of the plant, followed by further concentration and purification $[4,7]$. Figure 2 shows the main diterpene glycosides present in the $S$. rebaudiana aqueous extract.

Our recent studies on the preparation of porous nanomaterials revealed that the use of diterpene glycoside molecules from steviol as biological template favors the obtaining of metallic oxides with tubular morphology as nanorods [7] or nanofibers [4]. We are interested in understanding how these glycosides of kaurane diterpenoids control the nucleation and growth of inorganic materials, which favor the obtaining of various characteristic morphologies. It is for this reason that the employing of this ent-kaurane diterpenoids as biological templates for the preparation of porous nanomaterials is discussed on this communication. 


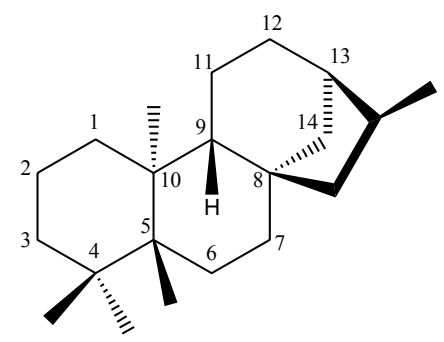

Figure 1. Carbon-skeleton of an ent-kaurane diterpene.
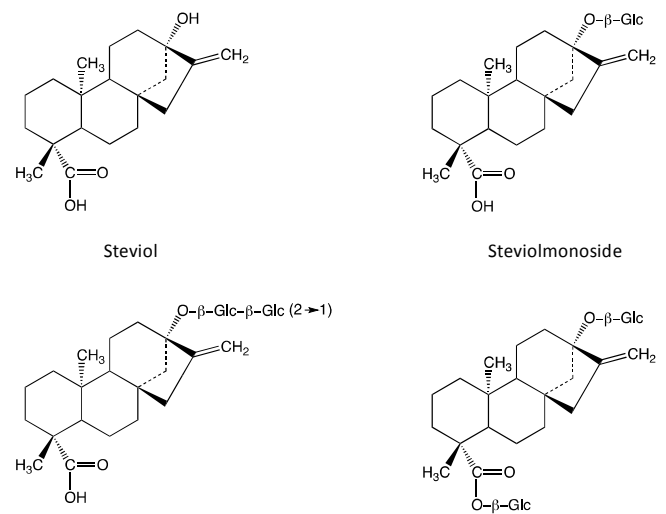

Steviolbioside
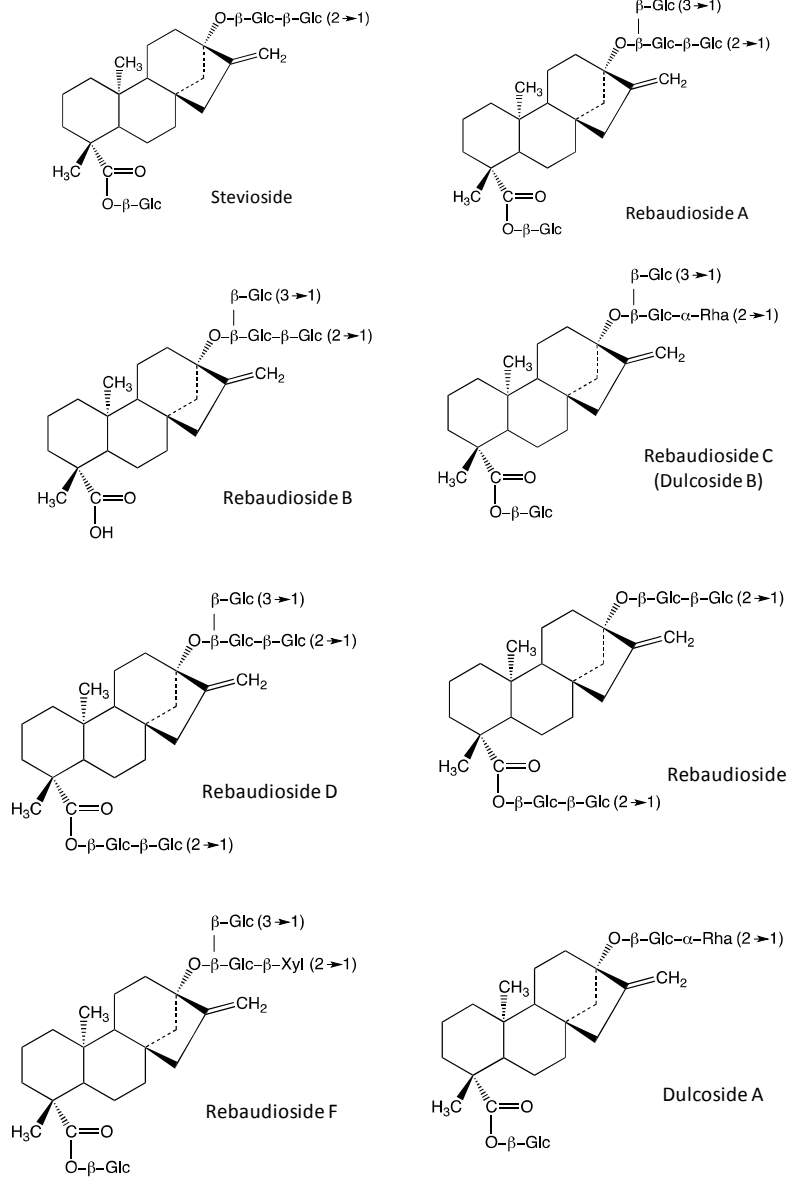

Figure 2. Diterpene glycosides from $S$. rebaudiana aqueous extract.

\section{Experimental}

\section{Preparation of Stevia rebaudiana Leaf Extract}

Portions of $1.2 \mathrm{~g}$ of leaves of $S$. rebaudiana were extracted with $50 \mathrm{~mL}$ of hot water $\left(65^{\circ} \mathrm{C}\right)$ for $3 \mathrm{~h}$, as described by Nishiyama et al. [5]. The crude extract was filtered through a Whatman qualitative filter paper No. 1. Finally the aqueous extract was drying at $60^{\circ} \mathrm{C}$.

Characterization of the extract was carried out by Fourier transform infrared (FTIR) recorded with a spectrophotometer Magna-IR 560 from Nicolet. The evaluation by transmission electron microscopy (HRTEM) was performed on a JEOL JEM-2100 microscope with $\mathrm{LaB}_{6}$ filament (accelerating voltage of $200 \mathrm{kV}$ ). The ${ }^{1} \mathrm{H}-\mathrm{NMR}$ was recorded on Bruker Advance $500 \mathrm{Mhz}$ spectrometer.

\section{Results and Discussion}

Recently, derivatives of isosteviol $((4,8,13)$-13-methyl16-oxo-17-norkaurane-18-carboxylic acid I), a diterpene of the kaurane series isolated from the hydrolysis products of glycosides present in S. rebaudiana plant, have been of particular interest because of the supramolecular structures that can form. The isosteviol has a rigid tetracyclic hydrocarbon skeleton and two polar reactive substituents (carboxyl and carbonyl groups at the $\mathrm{C} 4$ and C16 atoms respectively) arranged on the one side of the tetracyclic core (Figure 3). This attributed properties to be an ideal starting compound for constructing complex molecular structures capable of non-covalent binding of small and medium-size molecules (Figure 4) [6]. Previously, it has been shown that the isosteviol could form an individual molecular complex with aniline in crystal and solutions [6]. Alfonsov et al. demonstrated that the supramolecular structure of this complex looks like a double chiral helix cross-linked by aniline molecules (Figure 4). Additionally, it was concluded that the chiral spirals are united in bunches due to H-bonding between isosteviol molecules which belong to neighboring spirals. The formation of this complex was attributed to H-bonding between amine group of aniline and carboxyl group of isosteviol [8].

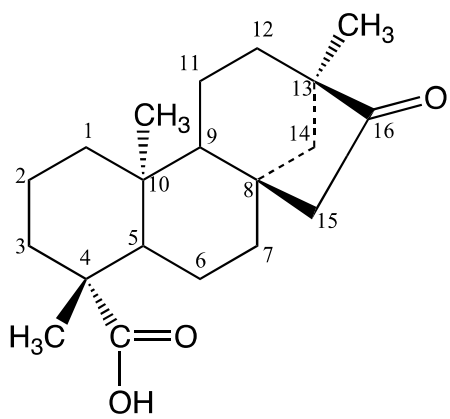

Figure 3. Chemical structure of isosteviol. 

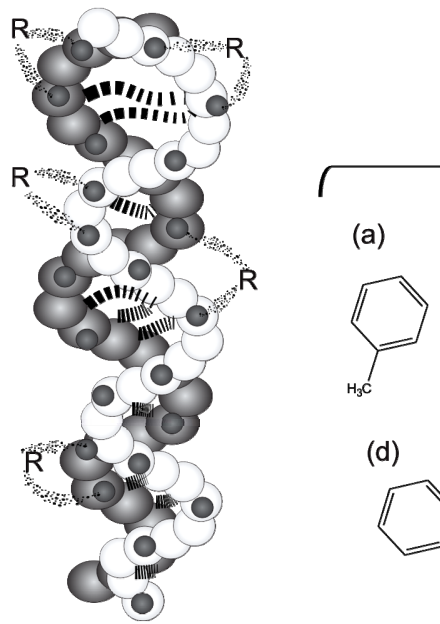

(d)

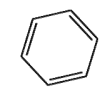

\section{$\mathrm{R}$}

(b)<smiles>Nc1ccccc1</smiles>

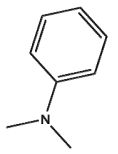

(e)

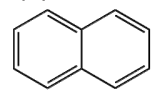

Figure 4. Supramolecular structures look like a double chiral helix cross-linked by aromatic molecules, e.g. (a) toluene; (b) aniline; (c) $\mathrm{N}, \mathrm{N}$-dimethylaniline; (d) benzene and (e) naphthalene.

The formation of these molecular complexes of isosteviol with other aromatic molecules also has been identified $[6,8]$. Surprisingly, it is observed that isosteviol can bind molecules, which have no possibility to H-bonding at all (benzene, naphthalene, toluene, xylenes, styrene, ethylbenzene, dimethylanilin). The formed complexes have been reported as isostructural in the space group $\mathrm{P}_{3} 2_{1} 2$ [9] (Figure 5).

According to this, it was established carrying out an HRTEM study of the $S$. rebaudiana dried extract, employed in the synthesis of nanosized metal oxides (Figure 6). HRTEM observations revealed that the glycosides of kaurane diterpenoids crystallize in cubic or tetragonal structures with diameters from 200 to $500 \mathrm{~nm}$. Recent studies based on the isosteviol derivatives and their analogs have attributed the formation of this crystal packing and supramolecular structures to the localization of the molecular regions with hydrophilic and hydrophobic properties (Figures 7 and 8). The morphology of the supramolecular structures formed depends on the ratio of volumes of hydrophilic and hydrophobic molecular fragments (Figure 8) [10]. To explain this phenomenon, the theory of microphase separation was used. This theory was developed previously for block-copolymer and liquid crystal systems [10]. In the HRTEM image can be seem nanostructures with different morphologies, which can be attributed to the different fragments both hydrophilic and hydrophobic of the glycosides present in the aqueous extract of the $S$. rebaudiana. The change of this ratio in the crystal also results in the modification of the morphology type of the structure, analogous to the modification of the supramolecular structure occurring in diblock-copolymers [10,11].

On the other hand, FTIR spectroscopic study of extract

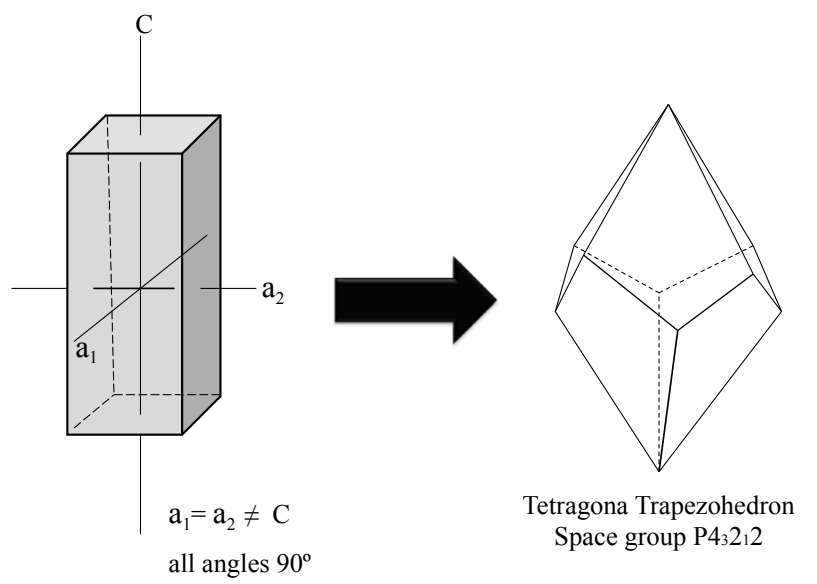

Figure 5. Crystal systems found in the formation of supramolecular structures of $S$. rebaudiana.

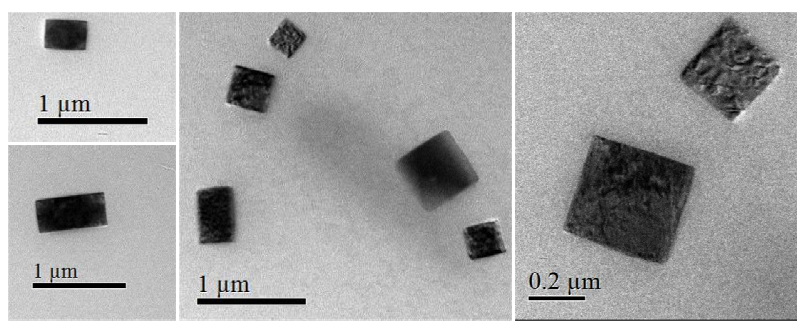

Figure 6. HRTEM image of the $S$. rebaudiana dried extract.

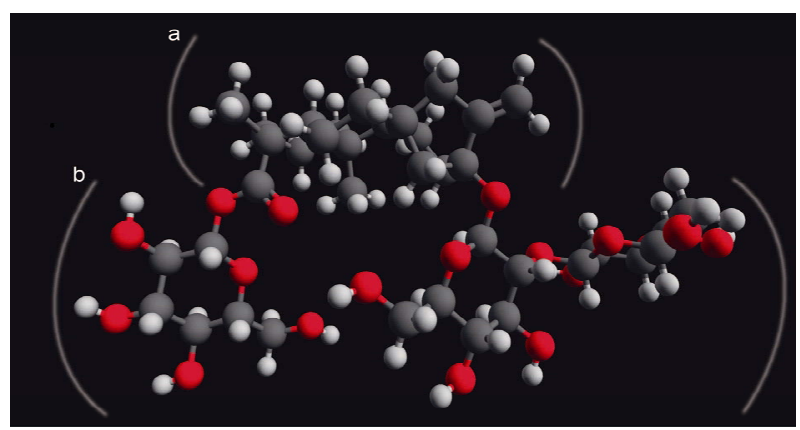

Figure 7. The distribution of the hydrophobic (a) and hydrophilic (b) fragments in the stevioside molecule.

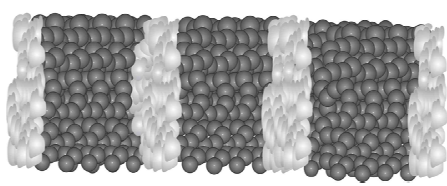

(a)

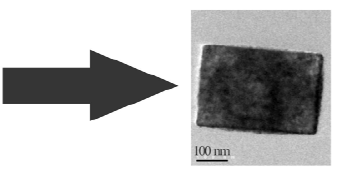

(b)
Figure 8. Model of (a) possible distribution of the hydrophilic (dark) and hydrophobic (light) fragments in a glycoside kaurane diterpenoids monocrystaland (b) HRTEM image of the glycoside of kaurane diterpenoids monocrystal from $S$. rebaudiana.

aqueous has been used to compare the changes in the functional groups with regard to the diterpenes isolated from S. rebaudiana glycosides (Table 1) [12]. 
Table 1. Fourier transform infrared (FTIR) spectroscopic study of extract aqueous and the diterpenes isolated from $S$. rebaudiana glycosides.

\begin{tabular}{|c|c|c|c|c|c|c|}
\hline & $\begin{array}{l}\text { OH Stretching } \\
\left(\mathrm{cm}^{-1}\right)\end{array}$ & $\begin{array}{c}\text { Alkane CH } \\
\text { Stretching }\left(\mathrm{cm}^{-1}\right)\end{array}$ & $C=O$ Stretching $\left(\mathrm{cm}^{-1}\right)$ & $\begin{array}{c}\mathrm{C}=\mathrm{C} \\
\text { Stretching }\left(\mathrm{cm}^{-1}\right)\end{array}$ & $\begin{array}{c}\text { C-O-C } \\
\text { Stretching }\left(\mathrm{cm}^{-1}\right)\end{array}$ & $\begin{array}{c}\mathrm{C}=\mathrm{C} \\
\text { Bending }\left(\mathrm{cm}^{-1}\right)\end{array}$ \\
\hline Steviol* Glycosides & $3319-3379$ & $2920-2938$ & $1720-1735$ & $1641-1662$ & $1054-1070$ & $870-894$ \\
\hline Extract aqueous & & 2929.44 & - & 1608 & 1075.61 & 817.54 \\
\hline
\end{tabular}

${ }^{*}$ FTIR spectral analysis of diterpene glycosides isolated from Stevia rebaudiana.

FTIR measurements of the stevia leaf extract aqueous are depicted in Figure 9. Different types of vibration may contribute to the broad band observed between 490 $\mathrm{cm}^{-1}$ and $817 \mathrm{~cm}^{-1}$ : bending vibrations of acetylenic C-H deformation, aliphatic $\mathrm{CH}_{3}$ deformation, olefinic cis$\mathrm{CH}=\mathrm{CH}$, as well as the out-of plane bending vibrations of the alcoholic bonds. The bands in $1385-1400 \mathrm{~cm}^{-1}$ range can be attributed to aliphatic $-\mathrm{CH}_{2}$ and $-\mathrm{CH}_{3}$ groups or bending modes of $\mathrm{O}-\mathrm{H}$ bonds.

The absorption band at $1730 \mathrm{~cm}^{-1}$ associated with $\mathrm{C}=\mathrm{O}$ bond stretching that frequently appear in the FTIR spectrum of the glycosides diterpenes "isolated" was not observed in the aqueous leaf extract from S. rebaudiana. This seems to indicate that the carbonyl groups participated in the assembly of the polyhedral structures, previously observed in HRTEM images, through interactions with $\mathrm{H}$ bound to neighboring spirals of ent-kaurane diterpenoids. This imply that the preparation of inorganic structures (metal oxides) using the leaf aqueous extract of S. rebaudiana as biological template cannot be carried out through the formation of a metal-carboxylate complex as in the case of other procedures of synthesis, which uses metal alkoxides and oligosaccharides (in acid medium). Another important point to mention is that the organic agent can only act controlling the hydrolysis of metal alkoxide if it contains in its structure active hydrogen atoms, hydroxyl groups, carboxyl, amide acid, imides, sulfonic acids, phenolic or ketoenolics groups [13]. This gave us a broad view of the applications that may have some vegetal extracts as biological templates in the synthesis of inorganic porous materials.

${ }^{1} \mathrm{H}-\mathrm{NMR}$ spectrum of Stevia leaf extract is depicted in Figure 10. The strong signal observed at $\delta=4.69 \mathrm{ppm}$ is due to $\mathrm{DHO}$ in $\mathrm{D}_{2} \mathrm{O}$. Signal at $\delta=0.74 \mathrm{ppm}$ could be due to aliphatic $\mathrm{CH}_{3}-\mathrm{C}$ groups, whereas the signals appearing between $\delta=1.0 \mathrm{ppm}$ and $\delta=1.297 \mathrm{ppm}$ are related to aliphatic $\mathrm{C}-\mathrm{CH}_{2}-\mathrm{C}$ and alkoxy $\mathrm{CH}_{3}-\mathrm{C}-\mathrm{O}$ groups, respectively [14]. It should be noted that any signal appears in the $\delta=10-12 \mathrm{ppm}$ range in which signals due to carboxylic acids are expected. This is in accordance with was mentioned above in the FTIR study.

\subsection{Preparation of Porous Inorganic Materials Using Aqueous Extract of $S$. rebaudiana}

Recently, we have described the synthesis of porous ma-

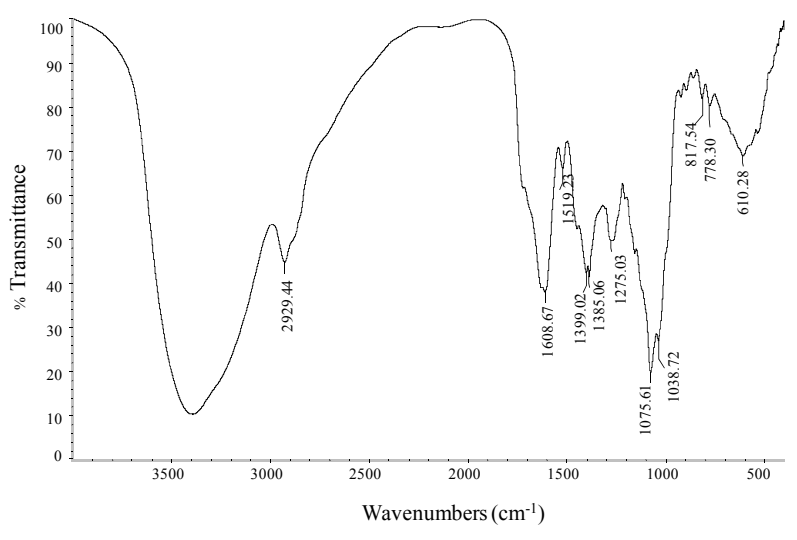

Figure 9. FTIR spectra of the stevia aqueous leaf extract.

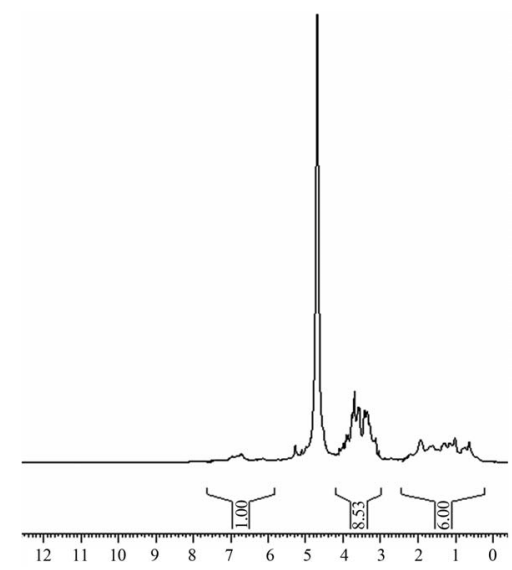

Figure 10. ${ }^{1} \mathrm{H}$-NMR spectrum of stevia aqueous leaf extract dissolved in $\mathrm{D}_{2} \mathrm{O}$.

terials using S. rebaudiana aqueous leaf extract $[4,7]$. The materials prepared by these method exhibit properties potentially useful for many applications in nanotechnology, in particular: high porosity, narrow pore size distributions and particle sizes ranging from $5-50 \mathrm{~nm}$. It is important to mention the case of the $\mathrm{TiO}_{2}$ prepared at room temperature in an aqueous medium, using titanium isopropoxide as precursor and the S. rebaudiana leaf extract as template [4]. The anatase phase of the synthesized $\mathrm{TiO}_{2}$ has a high surface area $\left(252 \mathrm{~m}^{2} \cdot \mathrm{g}^{-1}\right)$, diameter of $25-60 \mathrm{~nm}$, length of $100-150 \mathrm{~nm}$ and morphology of nanofibers (Figure 11). The observed morphology was similar to the morphology of alumina nanorods reported in our previous publication [7], which was also pre- 
pared employing the metal alkoxide. It is of interest to note that the prepared titania nanofibers are composed of many aggregates of nanocrystals that are added providing the resulting morphology (Figure 11). On the other hand, these aggregates are grouped together forming structures that correspond with the supramolecular arrangements, tetragonal and cubic, formed by the kaurane diterpenoids (Figure 12).

A proposal of the design of materials using the entkaurane diterpenoids as biological templates is shown in Figure 13. As you can see, the first step of the synthesis is the preparation of aqueous extract, which contains the biological template. Then, you must form a hybrid material together with the inorganic precursor. The formed structures could attach inorganic particles building tubular units (nanorods or nanotubes). Subsequently heat treatment will allow the removal of the biological template to finally obtain the metal oxide with tubular morphology.

\section{2. ent-Kaurane Diterpenoids from Different Plant Extracts}

The sweet ent-kaurane diterpene glycosides, which are considered in this contribution as potential biological templates, can be obtained from various plant extracts commonly used in the traditional herbal medicine. Among these is important to mention some of them. For example, phytochemical studies of the Wedelia trilobata showed that the plants of this genus are a rich source of ent-kaurane diterpenoids. The ent-kauranes recently isolated are shown in Figure 14 [15].

The study of the isolation and characterization of cussoracoside $C$ ent-kaurane diterpenoids, from Cussonia racemosa, represents a starting point for the investigation of other species such as Cussonia vantsilana from the same genus. From the leaves of the plant have been isolated a large amount of cussoracoside $C$ (Figure 15) together with four ent-kaurane diterpenoid glycosides, named cussovantosides A-D, respectively [16].

In addition, it has been also reported the ent-kaur16-en-18-oic acid, isolated from Croton antisyphiliticus root extract, showed in Figure 16 [17].

Finally, it is highlight the purification of the extract of dried seeds of Pharbitis nil Choisy (Convolvulaceae), which allowed obtaining six new ent-kaurane diterpene glycosides, pharbosides A-F [18]. There are some exam ples in Figure 17.

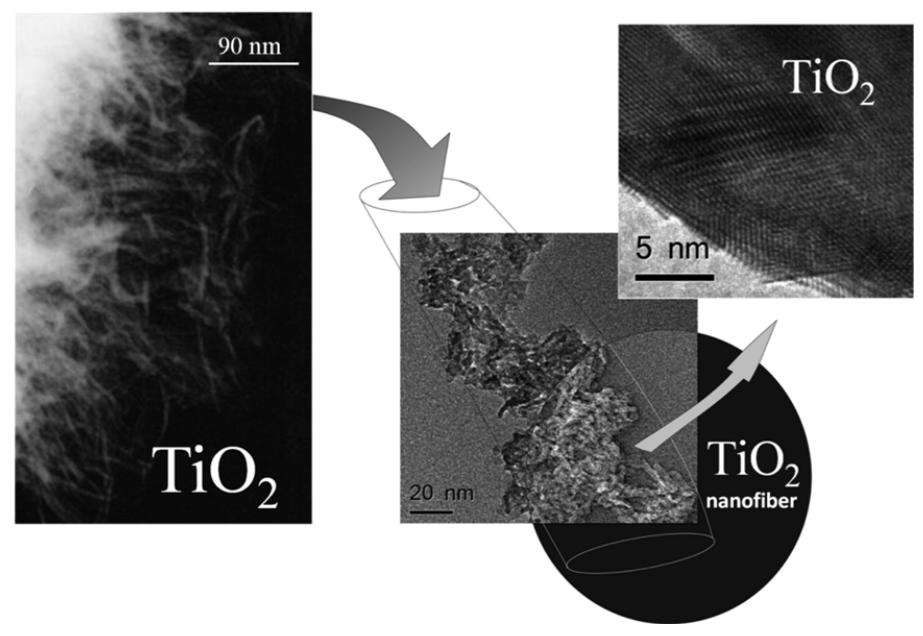

Figure 11. TEM and HRTEM of titania nanofibers.
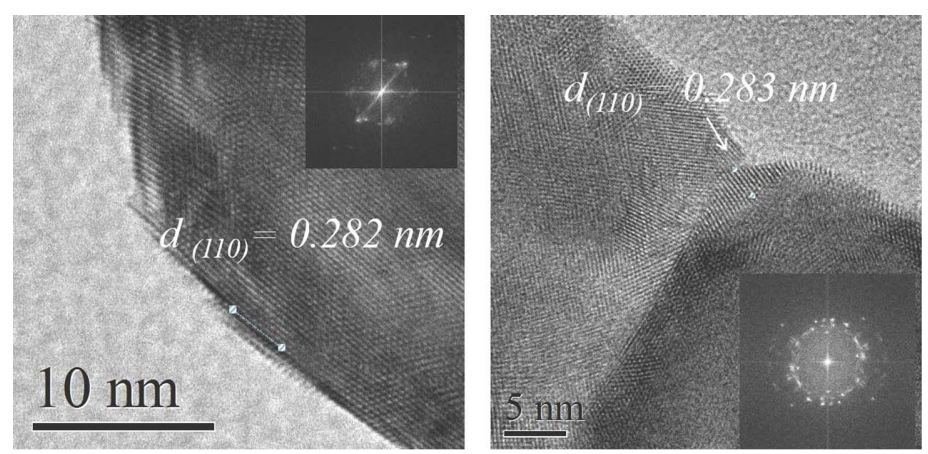

Figure 12. HRTEM image of the $\mathrm{TiO}_{2}$ prepared at room temperature in an aqueous medium, using titanium isopropoxide as precursor and the $S$. rebaudiana leaf extract as template. 


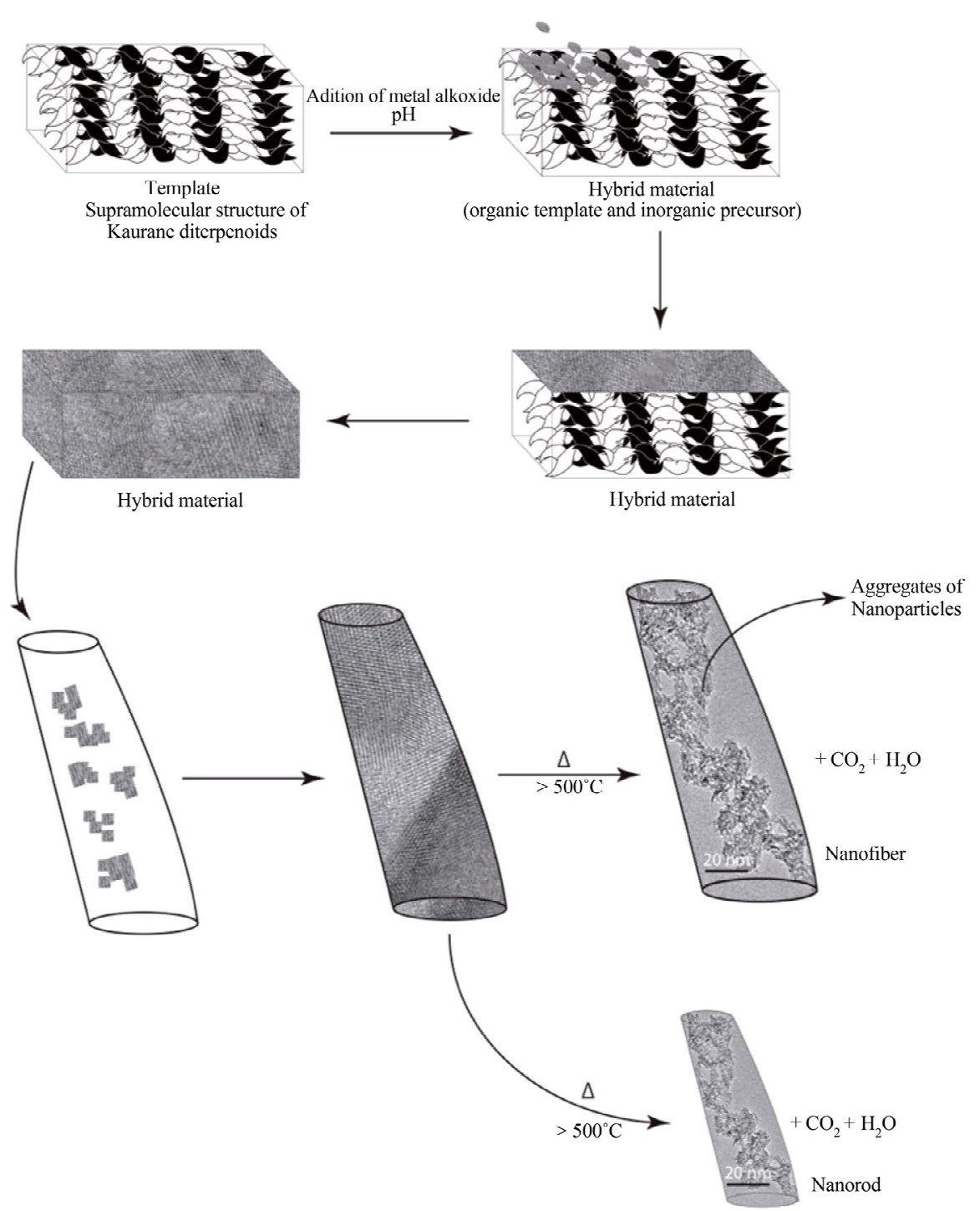

Figure 13. Scheme proposed for the design of materials using the kaurane diterpenoids as biological templates.

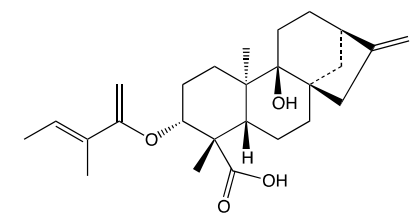

(1)

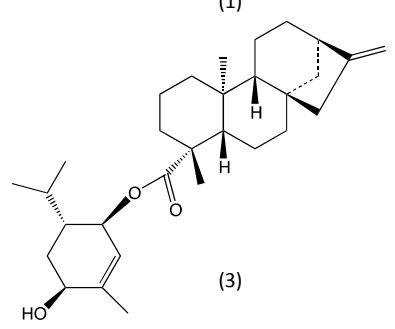

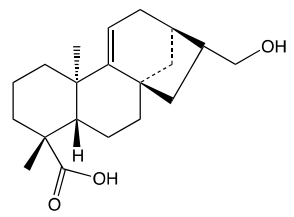

(2)

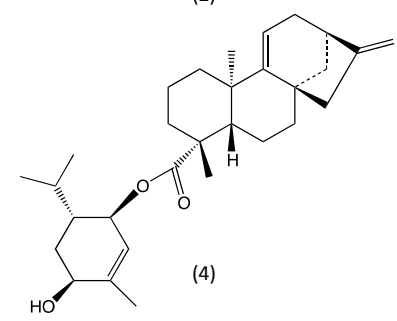

Figure 14. Molecular structure of ent-kauranes isolated of Wedelia trilobata: $3 \alpha$-tigloyloxypterokaurene L3 (1), ent-17hydroxykaura-9(11), 15-dien-19-oic acid (2) and wedelobatins $A$ and $B(3,4)$.

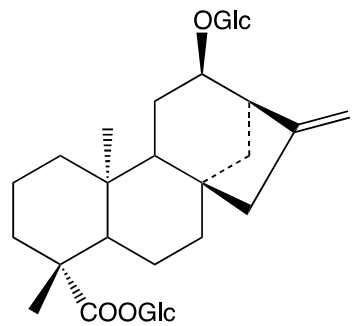

Figure 15. Molecular structure of cussoracoside $C$.

\section{Conclusion}

The ent-kaurane diterpenoids molecules are authentic biological templates with potential applications for the preparation of metal oxides. The HRTEM studies demonstrated that the glycosides of kaurane diterpenoids from S. rebaudiana form cubic or tetragonal crystal structures. The formation of metal oxide with tubular 


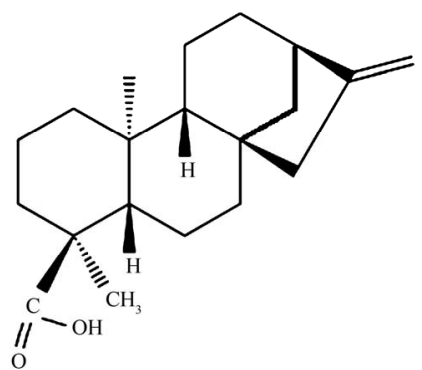

Figure 16. Molecular structure of ent-kaur-16-en-18-oic acid.

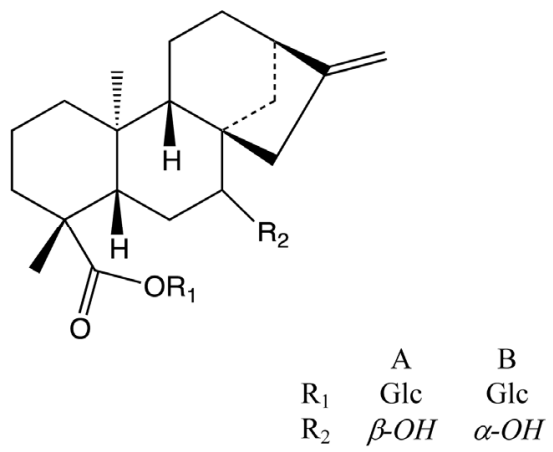

Figure 17. Molecular structure of pharbosides A and B.

morphology occurs as a result of the building of hybrid units of ent-kaurane diterpenoids molecules and the inorganic precursor, which is subjected to heat treatment at high temperature.

\section{Acknowledgements}

The authors are grateful to the Instituto Venezolano de Investigaciones Científicas (IVIC) project 1077 for financial support and Lic. Liz Cubillán for FTIR analysis.

\section{REFERENCES}

[1] E. Kataev, R. N. Khaybullin, R. R. Sharipova and I. Y. Strobykina, "ent-Kaurane Diterpenoids and Glycosides: Isolation, Properties, and Chemical Transformations," Review Journal of Chemistry, Vol. 1, No. 2, 2011, pp. 93160. http://dx.doi.org/10.1134/S2079978011010043

[2] P. Garcia, A. De Oliveira and R. Batista, "Occurrence, Biological Activities and Synthesis of Kaurane Diterpenes and Their Glycosides," Molecules, Vol. 12, No. 3, 2007, pp. 455-483. http://dx.doi.org/10.3390/12030455

[3] V. S. Prakash Chaturvedula, M. Upreti and I. Prakash, "Diterpene Glycosides from Stevia rebaudiana," Molecules, Vol. 16, No. 5, 2011, pp. 3552-3562. http://dx.doi.org/10.3390/molecules16053552

[4] M. Rodríguez, Á. B. Sifontes, F. J. Méndez, E. Cañizales, A. Mónaco, M. Tosta and J. L. Brito, "Synthesis and Characterization of Anatase $\mathrm{TiO}_{2}$ Nanofibers Using Stevia rebaudiana Leaf Aqueous Extract," In: A. Gayathri, Ed., Recent Research Developments in Material Sciences,
Research Signpost, Kerala, 2013, pp. 45-58.

[5] P. Nishiyama, M. Alvarez and L. G. E. Vieira, "Quantitative Analysis of Stevioside in the Leaves of Stevia rebaudiana by Near Infrared Reflectance Spectroscopy," Journal of the Science of Food and Agriculture, Vol. 59, No. 3, 1992, pp. 277-281.

http://dx.doi.org/10.1002/jsfa.2740590302

[6] V. A. Al'fonsov, O. V. Andreeva, G. A. Bakaleinik, D. V. Beskrovnyi, A. T. Gubaidullin, V. E. Kataev, G. I. Kovylyaeva, A. I. Konovalov, M. G. Korochkina, S. K. Latypov, I. A. Litvinov, R. Z. Musin and I. Y. Strobykina, "Chemistry and Structure of Diterpene Compounds of the Kaurane Series: VI. Isosteviol Esters," Russian Journal of General Chemistry, Vol. 73, No. 7, 2003, pp. 1119-1129. http://dx.doi.org/10.1023/B:RUGC.0000007623.48416.2b

[7] M. Rodríguez, Á. B. Sifontes, F. J. Méndez, Y. Díaz, E. Cañizales and J. L. Brito, "Template Synthesis and Characterization of Mesoporous $\gamma-\mathrm{Al}_{2} \mathrm{O}_{3}$ Hollow Nanorods Using Stevia rebaudiana Leaf Aqueous Extract," Ceramics International, Vol. 39, No. 4, 2013, pp. 4499-4506. http://dx.doi.org/10.1016/j.ceramint.2012.11.044

[8] V. A. Alfonsov, V. E. Kataev, O. V. Andreeva, D. V. Beskrovniy, G. I. Kovyljaeva, G. A. Bakaleynik, I. Y. Strobykina, I. A. Litvinov and A. I. Konovalov, "The Reception Activity of Isosteviol Isolated from the Plant Stevia rebaudiana Bertoni," Molecular Design and Synthesis of Supramolecular Architectures, Kazan, 27-31 August, 2002, p. 54.

[9] D. Beskrovnyy, A. Gubaidullin and I. Litvinov, "Crystal Structure of Isosteviol and Its Derivatives," $X X \mathrm{IUCr}$ Congress, Florence, 23-31 August 2005, p. C283.

[10] A. T. Gubaidullin, V. A. Mamedov, I. A. Litvinov, H. Ye and S. Tsuboi, "Synthesis and Comparative Analysis of Molecular and Supramolecular Structures of 4,8-Disubstituted 1,5-Dichloro-2,6-dioxotricyclo[5.1.0.0]octanes," Monatshefte für Chemie, Vol. 134, No. 9, 2003, pp. 12291240. http://dx.doi.org/10.1007/s00706-003-0028-y

[11] G. Wanka, H. Hoffmann and W. Ulbricht, "Phase Diagrams and Aggregation Behavior of Poly(oxyethylene)Poly(oxypropylene)-Poly(oxyethylene) Triblock Copolymers in Aqueous Solutions," Macromolecules, Vol. 27, No. 15, 1994, pp. 4145-4159. http://dx.doi.org/10.1021/ma00093a016

[12] V. S. P. Chaturvedula, "IR Spectral Analysis of Diterpene Glycosides Isolated from Stevia rebaudiana," Food and Nutrition Sciences, Vol. 03, No. 10, 2012, pp. 1467-1471. http://dx.doi.org/10.4236/fns.2012.310191

[13] B. K. Davison, "Organic Aluminium Compounds," US Patent No. 3184490, 1965.

[14] R. M. Silverstein, F. X. Webster and D. J. Kiemle, "Spectrometric Identification of Organic Compounds," 7th Edition, John Wiley \& Sons, 2005.

[15] B.-J. Ma, C.-N. Wen, Y. Gao, F.-C. Ren, F. Wang and J.-K. Liu, "ent-Kaurane Diterpenoids from the Plant Wedelia trilobata," Natural Products and Bioprospecting, Vol. 3, No. 3, 2013, pp. 107-111. http://dx.doi.org/10.1007/s13659-013-0029-4

[16] L. Harinantenaina, R. Kasai and K. Yamasaki, "ent-Kau- 
rane Diterpenoid Glycosides from a Malagasy endemic Plant, Cussonia vantsilana," Phytochemistry, Vol. 61, No. 4, 2002, pp. 367-372. http://dx.doi.org/10.1016/S0031-9422(02)00263-7

[17] S. Pereira, S. Taleb-Contini, J. Coppede, P. Pereira, B. Bertoni, S. Franca and A. M. Pereira, "An ent-KauraneType Diterpene in Croton antisyphiliticus Mart," Molecules, Vol. 17, No. 8, 2012, pp. 8851-8858. http://dx.doi.org/10.3390/molecules17088851

[18] K. H. Kim, S. U. Choi and K. R. Lee, "Diterpene Glycosides from the Seeds of Pharbitis nil," Journal of Natural Products, Vol. 72, No. 6, 2009, pp. 1121-1127. http://dx.doi.org/10.1021/np900101t 